\title{
The Laboratory Diagnosis of the Antiphospholipid Syndrome
}

\author{
Jasmina Ahluwalia $^{1} \cdot$ Sreejesh Sreedharanunni $^{1}$
}

Received: 1 July 2016/Accepted: 13 October 2016/Published online: 22 October 2016

(C) Indian Society of Haematology \& Transfusion Medicine 2016

\begin{abstract}
The Antiphospholipid Syndrome (APS) is classified based on the presence of both clinical and laboratory criteria. Both sets of criteria are subject to much review and intense research as it is becoming increasingly clear that no single test is specific for defining this autoimmune disorder. A number of leading international bodies have released guidelines in an attempt to improve the laboratory testing and reporting. The current review is an appraisal of some of the literature pertaining to the laboratory testing.
\end{abstract}

Keywords Antiphospholipid syndrome · Lupus anticoagulant $\cdot$ Anticardiolipin antibodies $\cdot$ Anti beta 2 Glycoprotein 1 antibodies - Laboratory tests

\section{Introduction}

The Antiphospholipid Syndrome (APS) also called the Hughes syndrome, in honour of the team that recognized the association of antiphospholipid antibodies and thrombosis, been has recognized as an important risk factor for thrombosis [1]. The syndrome is conveniently, but inaccurately, called antiphospholipid antibody syndrome, as the antibodies are not always directed against the phospholipid component. It is a systemic autoimmune condition encompassing primary APS, secondary APS, seronegative APS (SNAPS) and catastrophic APS (CAPS). While secondary APS occurs in patients with autoimmune disorders like systemic lupus erythematosus (SLE) or rheumatoid

Jasmina Ahluwalia

japgi@live.com; jasminapgi@gmail.com

1 Department of Hematology, Postgraduate Institute of Medical Education and Research, Chandigarh, India arthritis (RA); primary APS does not show such an association. CAPS is characterized by widespread thrombosis in a short time span, leading to multiorgan dysfunction. While the diagnosis of primary, secondary and CAPS is supported by laboratory parameters, SNAPS is a clinical diagnosis and is negative for lupus anticoagulant and antiphospholipid antibodies [2]. This review covers the recent guidelines, updates and some practical issues with the diagnosis of APS.

\section{Clinical Features of APS}

APS is classically associated with thrombosis and obstetric complications. Thrombosis can occur in both arterial and venous circulations. It can occur spontaneously or in the presence of other inherited or acquired provoking factors. Deep veins of lower extremities, pulmonary vessels and cerebral circulation are the common sites of thrombosis. Obstetric complications include intrauterine fetal death, recurrent abortions, abruptio placentae and toxemia of pregnancy.

APS is also associated with various other clinical features like thrombocytopenia, heart valve disease, cutaneous disorders (ulcers, livedo reticularis, superficial thrombophlebitis), renal disorders (APS nephropathy, renal artery stenosis), neurological dysfunction (migraine, epilepsy, cognitive impairment, dementia, transverse myelopathy), ocular problems (amaurosis fugax, retinal vessel thrombosis), and diffuse alveolar hemorrhage. These disorders are yet not considered in the classification criteria for the diagnosis of APS [3].

APS, though classically associated with thrombotic complications, occasionally leads to bleeding. The bleeding may be related to severe thrombocytopenia, platelet 
function disorders, factor VIII inhibitor, prothrombin deficiency and rarely to acquired deficiency of factors VII, $\mathrm{X}$ and XI. The co-existence of antiphospholipid antibodies with acquired prothrombin deficiency (lupus anticoagulanthypoprothrombinemia syndrome) occurs due to presence of binding antibodies against factor II leading to its clearance from the circulation [4].

\section{Pathogenesis}

APS is characterized by the presence of autoantibodies to wide variety of antigens, most importantly anionic phospholipid binding proteins like $\beta_{2}$ glycoprotein I ( $\left.\beta_{2} \mathrm{GPI}\right)$ [5] and prothrombin. These autoantibodies (lupus anticoagulant or LAC) have the ability to prolong clotting times in vitro and the commonly called antiphospholipid antibodies (aPL) [6]. Other antigenic targets include procoagulant proteins (high molecular weight kininogen, FV, FVII); anticoagulant proteins (protein $\mathrm{C}$ and $\mathrm{S}$ ); annexin A2 and A5; plasmin and vimentin [2]. $\beta_{2}$ GPI is the most important antigenic target. It is a glycoprotein with five domains - domain $\mathrm{I}$ is at the amino terminal while domain $\mathrm{V}$ occupies the carboxy terminal. It exists in two forms [7]. In the free form, domain $\mathrm{I}$ is bound to domain $\mathrm{V}$ to form a "coiled" configuration, masking the antigenic domain I from autoantibodies. Domain V binds to phospholipid to form a "fish hook" configuration. This exposes domain I to bind to the pathogenic autoantibodies [8].

A proportion of patients with APS have SLE or RA; however, the factors predisposing to the development of these autoantibodies is not yet clear. The pathogenesis of thrombosis is explained by a "two hit" model. According to this model, the first hit disrupts the endothelial integrity while the second hit potentiates thrombus formation. The mere presence of antibodies/antibody antigen complex cannot explain the clinical features in patients with APS. Many subjects with aPL antibodies remain asymptomatic. The first hit is provided by the factors like infection, recent surgery, smoking and other conditions that increase the oxidative stress and alters the vascular endothelial milieu. Oxidative stress alters $\beta_{2}$ GPI from a non-immunogenic, free thiol form to an immunogenic, oxidized form. Following the first hit, thrombus formation results from cumulative action of $\beta_{2}$ GPI-a $\beta_{2}$ GPI immune complexes on endothelium, platelets, monocytes, coagulation and anticoagulant proteins. Studies on animal models and humans have revealed various complex mechanisms involved in the thrombus formation. These include impaired function of endothelial nitric oxide synthase; activation of receptors on endothelial cells, platelets and monocytes (glycoprotein Iba, annexin A2, Toll like receptors); upregulation of tissue factor by aPL in monocytes, neutrophils and endothelial cells; disruption of the annexin A5 shield which usually inhibits the formation of procoagulant complexes; activation of complement $\mathrm{C} 3, \mathrm{C} 5$; and upregulation of toll like receptors TLR7 and TLR8 disrupting innate immunity. The activation of platelets, endothelium, monocytes, coagulation proteins and inflammatory cascade together with inhibition of fibrinolytic pathway and natural anticoagulation pathways (e.g.: protein C) leads to thrombosis [6]. Though, according to the widely accepted pathogenic model, it is believed that the autoantibodies directed against phospholipids are not pathogenic [9], current research reveals evidence for the pathogenic role of cofactor independent aPL and that the clinical studies do not support a dominant pathogenic role for anti $\beta_{2}$ GPI antibodies [10].

\section{Current Diagnostic Approach to APS}

The classification of APS is based on both clinical and laboratory features. The Sapporo criteria (1998) required one clinical and one laboratory criteria [11]. These criteria were later modified in the Sydney consensus meeting (2006). APS can be classified only in the presence of thrombotic (non inflammatory arterial, venous or small vessel thrombosis confirmed by imaging or histopathology) obstetric complications (death of one or more morphologically normal fetus at or beyond 10th week of gestation; one or more premature birth of normal fetus before 34th week due to eclampsia, pre-eclampsia or placental insufficiency; three or spontaneous abortions before 10th week provided other fetal or maternal causes are excluded) and persistent positivity (12 weeks apart) for LAC, anti- $\beta_{2}$ GPI or anti-cardiolipin (aCL) antibodies in moderate to high titers ( $>40$ GPL/MPL or $>99$ th percentile) [12]. Patients with non-criteria clinical features or antibodies (e.g.: $\operatorname{Ig} \mathrm{A}$ aCL/anti- $\beta_{2}$ GPI, antiphosphatidylserine antibodies (aPS), antiphosphatidylethanolamine antibodies (aPE), antibodies against prothrombin alone, antibodies against phosphatidyl serine prothrombin complex etc.) were to be classified into distinct groups like aPL associated nephropathy/thrombocytopenia/livedo reticularis etc. A significant number of cases of SNAPS may also be positive for other isotypes of antibodies which are not routinely tested, like anti domain I, IgA aCL or IgA a $\beta_{2}$ GPI [13].

\section{Issues with the Current Diagnostic Criteria of APS and Proposals for Updated Criteria}

The current criteria allow the diagnosis of APS even if one of the laboratory tests remains persistently positive [12]. Investigators have raised uncertainty in the definite 
diagnosis of APS on the basis of a single positive test. This is based on the lower incidence of first thromboembolic events in carriers with isolated LAC positivity compared to carriers with triple positivity (5.9 vs. $37 \%$ at 10 years) $[14,15]$. The risk of thrombosis was higher in carriers of two or three aPL antibodies when compared with those with single positivity [16]. Isolated LAC was not a risk factor for deep vein thrombosis in the Leiden thrombophilia study [17]. Further, it was shown that antibodies directed against a specific epitope (Gly40-Arg43) in the domain I of $\beta_{2}$ GPI (anti domain I antibodies) are responsible for LAC activity as well as thrombosis [18]. The significance of $\beta_{2}$ GPI independent LAC activity or aCL antibodies is under scrutiny. It is suggested that a specific subtype of antibodies (anti domain I type) responsible for thrombosis are detected when all the three tests are positive and it has been shown that triple positive patients have higher titer of anti domain I antibodies compared to double or single positive patients $[19,20]$. Based on these studies, it has been proposed to differentiate high risk triple positive APS patients from others [20] or to categorize APS into definite (triple positive), probable (dual positive) and possible or non-APS (single positive) [21]. Recent research has also questioned the evidence for suggesting a repeat testing at 12 weeks rather than at 6 weeks [22].

Recently, Pengo et al. proposed to classify APS into definite, probable/possible and uncertain categories. A diagnosis of APS can be definite if the patient satisfies the 2006 International consensus criteria Sydney with positivity for LAC, aCL as well as anti- $\beta_{2}$ GPI antibodies. They are at high risk for recurrent thrombosis or pregnancy morbidity. These patients may not require repeat testing after 12 weeks as the results are highly likely to be persistent. APS is "probable" if only two of the laboratory tests are positive. They are often negative for LAC as the titer of aCL or $\beta_{2}$ GPI antibodies are not high enough to induce LAC activity in the plasma. APS is considered "uncertain" if only one of the tests turns positive. Low titer single test positivity may be significant in obstetric cases unlike thrombosis [23].

\section{Patients to be Tested for APS}

Because of risk of false positivity, asymptomatic individuals should not be tested to identify those at risk of thrombosis. The pretest probability (strong indication) for a positive test is "high" for young individuals $<50$ years with unprovoked arterial or venous thrombosis, patients with thrombosis at unusual sites, late pregnancy loss and in those with thrombosis or pregnancy morbidity associated with autoimmune diseases. The indication is "moderate" in those with incidentally detected prolonged aPTT, recurrent early pregnancy loss or provoked VTE in young individuals. The risk is "low" in elderly patients with thrombosis [24]. Clinical discretion is required in patients with SLE or other autoimmune disorders [25].

\section{The "Available" and the "Recommended Tests"}

Laboratory tests are broadly classified into the clot based LAC (which detects the in vitro inhibitory activity of aPL antibodies) and solid phase assays. Laboratory testing and reporting should follow one of the recent guidelines [24, 26-28]. The LAC can be tested by a variety of testsdiluted Russell Viper Venom time (dRVVT), aPTT with silica as an activator (Silica Clotting time), Kaolin clotting time (KCT), dilute prothrombin time (dPT), ecarin clotting time (ECT) and textarin clotting time. It is generally accepted that a single test will not be able to identify clinically significant LAC in all patients. Hence at least two tests are to be performed. ISTH and other guidelines recommend dRVVT as the first choice [24, 27, 28]. The International Society on Thrombosis and Hemostasis (ISTH) recommends an aPTT with low phospholipids and silica activator as second choice. Other tests like KCT, ECT and dPT are not recommended. The British Committee for Standards in Hematology (BCSH) guidelines include use of aPTT with proven LAC sensitivity, modified aPTT or $\mathrm{dPT}$ as a second choice. The Clinical and Laboratory Standards Institute (CLSI) 2014 recommends a lupus responsive aPTT as the second choice and does not exclude the utility of other tests. While the ISTH guideline is relatively clear about the second choice, other guidelines are more generalized advocating reagents or test that are "lupus sensitive" or "lupus responsive" [24, 27, 28]. aPL antibodies which can be tested include IgG, IgM and IgA antibodies to the antigens already referred to. Among these, currently it is recommended to test only for IgG and IgM antibodies to $\beta_{2}$ GPI and aCL [26]. There are issues with the standardization of the other tests and data on the clinical utility is limited. The anti domain I antibodies, which have been shown to correlate with thrombosis and obstetric complications are promising new candidates [2, 26]. Using an automated anti-domain I assay, high titres with this assay was found to be associated with triple positive APS and thrombosis [29], however this testing did not add significant diagnostic value to the existing panel in a recent report.

\section{Need for Doing Multiple Tests}

None of the available tests are able to identify all the patients with APS. There are issues with the standardization and accuracy of each test. Positivity for a single test in 
isolation does not have much significance compared to the positivity for multiple tests.

\section{Timing of the Test and Tests that can be Done if the Patient is on Anticoagulants}

Preferably, LAC testing is avoided during acute episode. Elevated FVIII levels can lead to false negative results while elevated $\mathrm{C}$ reactive proteins can lead to false positive screening test results [8]. Most anticoagulants interfere with LAC testing. In patients on vitamin $\mathrm{K}$ antagonists (VKA) the INR may be performed. If it is $<1.5$, LAC can be done on undiluted plasma and if between 1.5 and 3 , then LAC can be tested on a 1:1 mixture of patient and normal pooled plasma [24]. BCSH and CLSI guidelines recommend testing of LAC screen and confirm on 1:1 mixture irrespective of INR with the caution that a negative result in such mixtures does not exclude LAC. BCSH and CLSI also support the use of Taipan snake venom test (TSVT) (screen) combined with ECT or platelet neutralization procedure (confirm) [27, 28]. The experience with these tests is limited. Another strategy is to temporarily withhold VKA or to bridge with low molecular weight heparin (LMWH) till the test is done [27]. In case of Unfractionated Heparin (UFH), it is preferable to avoid testing [27], even though the heparin neutralizers in the commercial reagents, may quench heparin to a certain extent. LMWH usually do not interfere with DRVVT. The LAC should be avoided while on direct thrombin inhibitors, while TSVT or ECT are useful in patients on direct Xa inhibitors [28]. Solid phase ELISA tests for aCL or $\beta_{2}$ GPI antibodies can be done at any time as they are unaffected by anticoagulants.

\section{Sample Collection and Preanalytical Considerations}

Extreme care is to be taken in the pre analytical stage to avoid activation of the sample, loss of coagulant proteins due to their natural degradation, contamination by anticoagulants and by platelet phospholipid. The sample is to be collected into $0.109 \mathrm{~mol} / \mathrm{L}$ trisodium citrate by minimal trauma and plasma is rendered platelet poor $(<1000 / \mathrm{L})$ preferably by double centrifugation technique and not by ultracentrifugation or filtration techniques. The plasma may be tested immediately or frozen at $-70{ }^{\circ} \mathrm{C}$ as quickly as possible and stored. Repeated freezing and thawing is to be avoided. Thawing is to be done at $37{ }^{\circ} \mathrm{C}$ for $5 \mathrm{~min}$ to avoid formation of cryoprecipitate $[24,27,28]$. Serum or doublecentrifuged platelet poor plasma is be suitable for ELISA tests for ACA and aß2GP1 antibodies. Samples can be stored at $2-8{ }^{\circ} \mathrm{C}$ for $2-3$ days or at $-20{ }^{\circ} \mathrm{C}$ or below for longer durations [26].

\section{Lupus Anticoagulant Testing (Analytical Considerations and Reporting)}

Testing of PT, aPTT and thrombin time (TT) (to exclude undiagnosed coagulopathy or anticoagulant therapy) should ideally precede LAC testing. dRVVT and SCT are done in three steps-screening, confirmation and mixing study. Screening is done using a reagent with low phospholipid levels, which makes it sensitive to LAC and prolongs the clotting time beyond the normal reference range. In the confirmatory assay, the same reagent with higher phospholipid content is used. This is to confirm the phospholipid dependent inhibitory activity of the LAC. The higher phospholipid content helps to neutralize the antibody and brings the clotting time to normal. The screening and confirmatory tests performed using the same reagents on a 1:1 mixture with normal pooled plasma also helps to confirm the presence of an inhibitor especially in the patients with prolonged screening and confirmatory tests, provided coagulopathies and presence of anticoagulants are excluded. The guidelines differ in their recommendations regarding the order of performance of confirmatory and mixing studies. LAC should always be tested by two parallel assays. No single test is sensitive enough to detect all cases. Patients who are positive for both tests are likely to remain persistently positive [30].

For screen, mixing study and confirm tests, a reference range has to be established locally by performing screen, mix (1:1 mix of normal controls with normal pooled plasma) and confirm tests on ideally $>120$ subjects or at least 40 subjects; or a previously established reference can be validated on 40-60 samples. 99th percentile (2.3 SD) or 97.5th percentile (2 SD) is used to establish cut offs. 99th percentile reduces the chances of false positivity but at the same time reduces sensitivity [24, 27, 28]. Different approaches can followed in LAC testing (Fig. 1).

A screening test (e.g.: dRVVT screen and SCT screen) is performed, if positive ( $>99$ th percentile/ $>97.5$ th percentile), a mixing study follows. If the mixing study suggests an inhibitor [clotting time of 1:1 mix of patient plasma with normal pooled plasma $>$ cutoff or index of circulating anticoagulant calculated as (clotting time of mixture-clotting time of normal pooled plasma) $\div$ clotting time of patient plasma $\times 100>$ cutoff], the phospholipid dependence is confirmed using a reagent with higher concentration of bilayer or hexagonal (II) phase phospholipid (dRVVT confirm and SCT confirm). The confirm test is considered positive if the percentage correction (screen-confirm)/screen 100 is $>$ cut off value or 
Fig. 1 Approaches in LAC testing

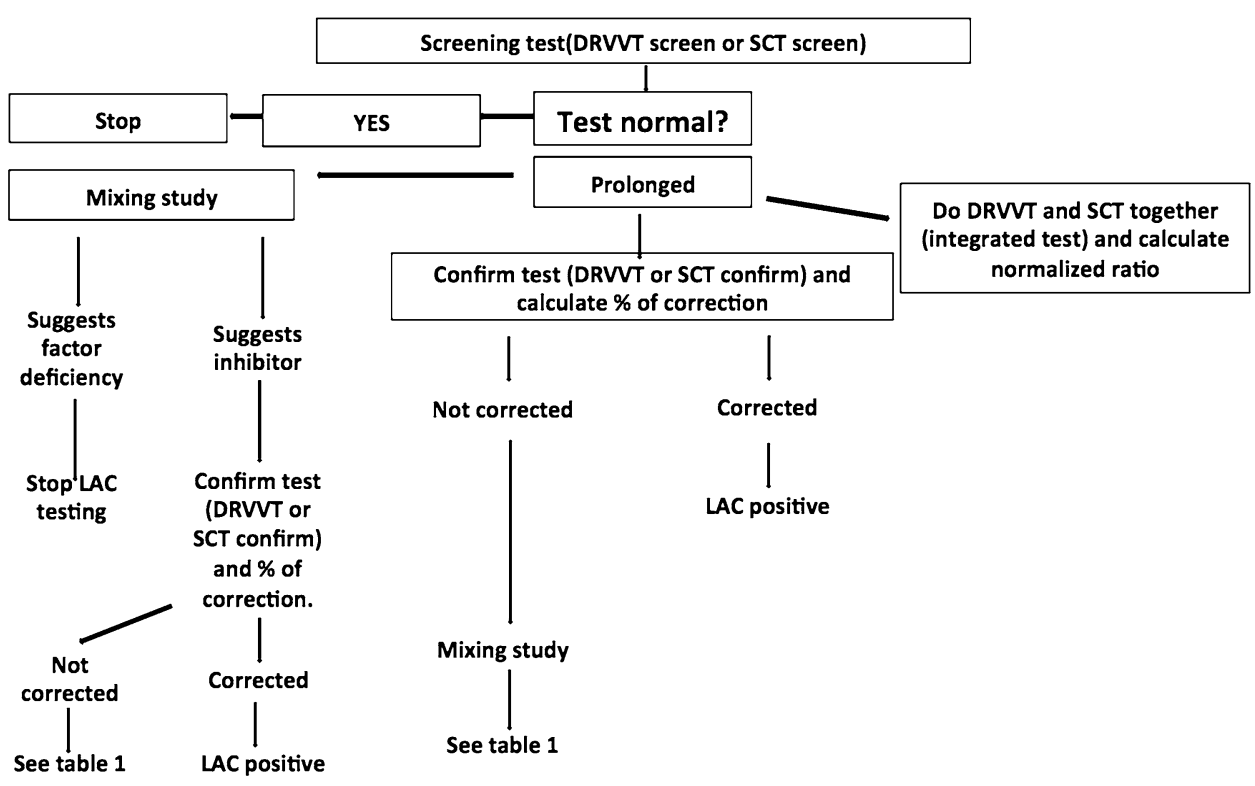

Table 1 Possible interpretations of screening, confirm and mixing studies

\begin{tabular}{|c|c|c|c|}
\hline Screen time & Confirm time & Clotting time of mix & Interpretation \\
\hline Normal & Not done & Not done & Negative for LAC \\
\hline Prolonged & Corrected/high normalized ratio & Prolonged & Positive for LAC \\
\hline Prolonged & Corrected/high normalized ratio & Corrected & $\begin{array}{l}\text { Does not exclude LAC } \\
\text { Probably dilution effect }\end{array}$ \\
\hline Prolonged & Not corrected but high normalized ratio & Prolonged & $\begin{array}{l}\text { Positive for LAC } \\
\text { Probably potent LAC or LAC with other inhibitors }\end{array}$ \\
\hline Prolonged & Not corrected and normal normalized ratio & Prolonged & $\begin{array}{l}\text { Other inhibitors } \\
\text { Check for anticoagulant }\end{array}$ \\
\hline Prolonged & Not corrected and normal normalized ratio & Corrected & Factor deficiency \\
\hline
\end{tabular}

normalized ratio calculated as (screen of patient/screen of normal $) \div$ (confirm of patient/confirm of normal) $>$ locally derived cut off [24, 27]. CLSI 2014 recommends screening followed by a confirm test and mixing study [28]. A mixing study helps to identify an inhibitor, however it can be falsely negative due to dilution of antibodies. Therefore, if screen and confirm tests are suggestive of LAC, a negative mixing study still does not exclude its presence. In some cases, the LAC might be very potent resulting in a failure of correction with confirmatory reagents, mixing study is potentially helpful in such situations to confirm the presence of inhibitor. In a given situation, mixing study may be omitted if the results of screen and confirm are clear-cut and the presence of coagulation factor deficiency or anticoagulants are excluded by normal aPTT (with lupus insensitive reagents), PT and TT. Mixing study may be performed in all cases or decided on case-tocase basis [28]. Table 1 shows the interpretation of some combinations observed.
Another approach is to perform integrated tests. Both the screen and confirm tests are performed simultaneously in all cases (e.g.: dRVVT screen and confirm, SCT screen and confirm). A normalized ratio is calculated for each test to decide positivity. Calculation of the normalized ratios helps to overcome the inter- and intra-assay variability arising from issues with analyzer, reagent and operator [24, 28]. LAC is reported as positive or negative with the quantitative values and ratios. In cases where the interpretation is not clear, a repeat test is advised after a few days or weeks [24].

\section{aCL and $\mathbf{a} \beta_{2}$ GPI Antibodies (Analytical Considerations and Reporting)}

aCL and $a \beta_{2}$ GPI antibodies are commonly tested by commercial, solid phase ELISA kits. Fluid phase immunoassays are insensitive to these antibodies, because 
in fluid state, the $\beta_{2}$ GPI molecule assumes a closed loop configuration hiding the cryptic epitope on domain I from binding to antibodies [8]. ELISA tests have high turnaround time in low volume laboratories. The experience with automated assay systems are limited [31]. The inconsistencies in the exposure of critical areas of domain I of $\beta_{2}$ GPI on ELISA plates is probably responsible for some of the interlaboratory variability in the performance of $\mathrm{a} \beta_{2}$ GPI ELISA [8]. IgG and IgM isotypes are measured. Measurement of a $\beta_{2}$ GPI antibodies should be done using $\beta_{2}$ GPI antigen of human origin attached to a negatively charged plate. Serum samples or doubly centrifuged platelet poor plasma can be used. The instructions of the manufacturer should be followed. Icteric, hemolytic and lipemic samples are better avoided. Calibrators (at least 6 points) and internal quality control samples should be included in each run. The calibrators, quality controls and patient sample should ideally be run in duplicate and the coefficient of variation $(\mathrm{CV})$ should be $<15$ and $<10 \%$ in manual and automated platforms respectively. Cut off values (99th percentile) should be determined locally using 120 normal healthy donors. If this is not possible then the manufacturers' cut off is validated using samples from at least 20 healthy subjects. Currently there is no international unit uniformly employed to report the results. The unit depends on the calibrator used. If Louisville standards are used, aCL is expressed in GPL (G Phospholipid) or MPL (M Phospholipid) units. a $\beta_{2}$ GPI antibodies are reported in arbitrary units. A cut-off value of $>99$ th percentile (moderate to high titer) is more accurate than $>40$ GPL/MPL cut off. All positive tests have to be confirmed by repeating the test after an interval of 12 weeks $[8,26]$.

In our experience, the IgG $\beta 2 \mathrm{GPI}$ is the most common antibody positive in patients with thrombotic APS [31]. Triple positive APS is uncommon. While triple positivity at first visit had highest specificity and positive predictive value for the diagnosis of APS, anti $\beta_{2}$ GPI antibodies have the highest sensitivity and negative predictive value.

Detection of the aCL and $\beta_{2}$ GPI antibodies by chemiluminescence technology (BIOFLASH ${ }^{\circledR}, \mathrm{ACL} \mathrm{AcuStar}^{\circledR}$ ) shows good sensitivity and specificity, compares favourably with the ELISA, has rapid turnaround times and lower $\mathrm{CV}$ of testing making this a value addition to high volume laboratories [32, 33].

\section{Risk of Thrombosis Assessment-Global Antiphospholipid Score (GAPSS)}

A scoring system has been developed to predict the risk of thrombosis or pregnancy lossin patients with APS taking into account the aPL profile, conventional cardiovascular risk factors and the profile of autoimmune antibodies. The parameters and scores included in GAPSS are hyperlipidemia (score 3), arterial hypertension (score 1), IgG/IgM aCL (score 5), IgG/IgM a $\beta_{2}$ GPI (score 4), IgG/IgM antiphosphatidyl serine (aPS)/anti prothrombin (score 3), and LAC (score 4). A GAPSS value of $\geq 10$ or $\geq 11$ was reported to have the best diagnostic accuracy for thrombosis or pregnancy loss [34-36].

\section{Conclusions}

The testing of APS antibodies involves a number of laboratory tests of considerable complexity at the preanalytical, analytic and post analytical stages. Reporting laboratories have a choice of guidelines to work with. Participation in quality assurance exercises is important to assess performance. There is scope for standardization to ensure uniformity in the existing methods and newer technologies. Correlation of test results with clinical outcome would have a defining role in the choice of tests and techniques that laboratories adopt.

\section{Compliance with Ethical Standards}

Conflict of interest Dr Jasmina Ahluwalia and Dr Sreejesh Sreedharanunni have no conflict of interest to declare.

Ethical Approval This article does not contain any studies with human participants performed by any of the authors. This article does not contain any studies with animals performed by any of the authors.

\section{References}

1. Khamashta M, Hughes GRV (1999) Hughes syndrome: a short history of the antiphospholipid syndrome. In: Schoenfeld Y (ed) The decade of autoimmunity. Elsevier Science BV

2. Nayfe R, Uthman I, Aoun J, SaadAldin E, Merashli M, Khamashta MA (2013) Seronegative antiphospholipid syndrome. Rheumatology (Oxf Engl) 52:1358-1367

3. Meroni PL, Chighizola CB, Rovelli F, Gerosa M (2014) Antiphospholipid syndrome in 2014: more clinical manifestations, novel pathogenic players and emerging biomarkers. Arthritis Res Ther 16:1-14

4. Forastiero R (2012) Bleeding in the antiphospholipid syndrome. Hematology (Amst Neth) 17(Suppl 1):153-155

5. de Groot PG, Urbanus RT, Derksen RHWM (2012) Pathophysiology of thrombotic APS: Where do we stand? Lupus 21:704-707

6. Giannakopoulos B, Krilis SA (2013) The pathogenesis of the antiphospholipid syndrome. N Engl J Med 368:1033-1044

7. de Groot PG, Meijers JCM (2011) $\beta(2)-$ Glycoprotein I: evolution, structure and function. J Thromb Haemost JTH 9:1275-1284

8. Krilis SA, Giannakopoulos B (2014) Laboratory methods to detect antiphospholipid antibodies. Hematol Am Soc Hematol Educ Program 2014:321-328

9. de Groot PG, Urbanus RT (2012) The significance of autoantibodies against $\beta 2$-glycoprotein I. Blood 120:266-274 
10. Lackner KJ, Müller-Calleja N (2016) Pathogenesis of the antiphospholipid syndrome revisited: time to challenge the dogma. J Thromb Haemost JTH 14:1117-1120

11. Wilson WA, Gharavi AE, Koike T, Lockshin MD, Branch DW, Piette JC et al (1999) International consensus statement on preliminary classification criteria for definite antiphospholipid syndrome: report of an international workshop. Arthritis Rheum 42:1309-1311

12. Miyakis S, Lockshin MD, Atsumi T, Branch DW, Brey RL, Cervera $\mathrm{R}$ et al (2006) International consensus statement on an update of the classification criteria for definite antiphospholipid syndrome (APS). J Thromb Haemost JTH 4:295-306

13. Cousins L, Pericleous C, Khamashta M, Bertolaccini ML, Ioannou Y, Giles I et al (2015) Antibodies to domain I of $\beta$-2-glycoprotein I and $\operatorname{IgA}$ antiphospholipid antibodies in patients with "seronegative" antiphospholipid syndrome. Ann Rheum Dis 74:317-319

14. Pengo V, Testa S, Martinelli I, Ghirarduzzi A, Legnani C, Gresele P et al (2015) Incidence of a first thromboembolic event in carriers of isolated lupus anticoagulant. Thromb Res 135:46-49

15. Pengo V, Ruffatti A, Legnani C, Testa S, Fierro T, Marongiu F et al (2011) Incidence of a first thromboembolic event in asymptomatic carriers of high-risk antiphospholipid antibody profile: a multicenter prospective study. Blood 118:4714-4718

16. Mustonen P, Lehtonen KV, Javela K, Puurunen M (2014) Persistent antiphospholipid antibody (aPL) in asymptomatic carriers as a risk factor for future thrombotic events: a nationwide prospective study. Lupus 23:1468-1476

17. de Groot PG, Lutters B, Derksen RHWM, Lisman T, Meijers JCM, Rosendaal FR (2005) Lupus anticoagulants and the risk of a first episode of deep venous thrombosis. J Thromb Haemost JTH 3:1993-1997

18. de Laat B, Derksen RHWM, Urbanus RT, de Groot PG (2005) IgG antibodies that recognize epitope Gly40-Arg43 in domain I of beta 2-glycoprotein I cause LAC, and their presence correlates strongly with thrombosis. Blood 105:1540-1545

19. Banzato A, Pozzi N, Frasson R, De Filippis V, Ruffatti A, Bison $E$ et al (2011) Antibodies to domain I of $\beta(2)$ Glycoprotein I are in close relation to patients risk categories in antiphospholipid syndrome (APS). Thromb Res 128:583-586

20. Pengo V, Banzato A, Denas G, Jose SP, Bison E, Hoxha A et al (2013) Correct laboratory approach to APS diagnosis and monitoring. Autoimmun Rev 12:832-834

21. Forastiero R (2014) Multiple antiphospholipid antibodies positivity and antiphospholipid syndrome criteria re-evaluation. Lupus 23:1252-1254

22. Park SH, Jang S, Park C-J, Chi H-S (2016) Clinical application of revised laboratory classification criteria for antiphospholipid antibody syndrome: Is the follow-up interval of 12 weeks instead of 6 weeks significantly useful? BioMed Res Int 2016:2641526

23. Pengo V, Denas G, Padayattil SJ, Zoppellaro G, Bison E, Banzato A et al (2015) Diagnosis and therapy of antiphospholipid syndrome. Pol Arch Med Wewn 125:672-677
24. Pengo V, Tripodi A, Reber G, Rand JH, Ortel TL, Galli M et al (2009) Update of the guidelines for lupus anticoagulant detection. Subcommittee on Lupus anticoagulant/antiphospholipid antibody of the Scientific and Standardisation Committee of the International Society on Thrombosis and Haemostasis. J Thromb Haemost JTH 7:1737-1740

25. Rand JH, Wolgast LR (2012) Dos and don'ts in diagnosing antiphospholipid syndrome. Hematol Am Soc Hematol Educ Program 2012:455-459

26. Devreese KMJ, Pierangeli SS, de Laat B, Tripodi A, Atsumi T, Ortel TL et al (2014) Testing for antiphospholipid antibodies with solid phase assays: guidance from the SSC of the ISTH. J Thromb Haemost 12:792-795

27. Keeling D, Mackie I, Moore GW, Greer IA, Greaves M (2012) British Committee for Standards in haematology. Guidelines on the investigation and management of antiphospholipid syndrome. Br J Haematol 157:47-58

28. Moore GW (2014) Recent guidelines and recommendations for laboratory detection of lupus anticoagulants. Semin Thromb Hemost 40(2):163-171

29. De Craemer AS, Musial J, Devreese KM (2016) Role of antidomain 1- $\beta 2$ glycoprotein I antibodies in the diagnosis and risk stratification of antiphospholipid syndrome. J Thromb Haemost 14(9):1779-1787. doi:10.1111/jth.13389

30. Simmons DP, Herskovits AZ, Battinelli EM, Schur PH, Lemire SJ, Dorfman DM (2016) Lupus anticoagulant testing using two parallel methods detects additional cases and predicts persistent positivity. Clin Chem Lab Med. doi:10.1515/cclm-2015-0790. pii: /j/cclm.ahead-of-print/cclm-2015-0790/cclm-2015-0790.xml

31. Ahluwalia J, Sreedharanunni S, Kumar N, Masih J, Bose SK, Varma $N$ et al (2016) Thrombotic primary antiphospholipid syndrome: the profile of antibody positivity in patients from North India. Int J Rheum Dis 19(9):903-912

32. Meneghel L, Ruffatti A, Gavasso S, Tonello M, Mattia E, Spiezia L et al (2015) The clinical performance of a chemiluminescent immunoassay in detecting anti-cardiolipin and anti- $\beta 2$ glycoprotein I antibodies. A comparison with a homemade ELISA method. Clin Chem Lab Med 53:1083-1089

33. Oku K, Amengual O, Kato M, Bohgaki T, Horita T, Yasuda S et al (2016) Significance of fully automated tests for the diagnosis of antiphospholipid syndrome. Thromb Res 146:1-6

34. Sciascia S, Sanna G, Murru V, Roccatello D, Khamashta MA, Bertolaccini ML (2015) The global anti-phospholipid syndrome score in primary APS. Rheumatology (Oxf Engl) 54:134-138

35. Sciascia S, Sanna G, Murru V, Roccatello D, Khamashta MA, Bertolaccini ML (2013) GAPSS: the Global Anti-Phospholipid Syndrome Score. Rheumatology (Oxf Engl) 52:1397-1403

36. Sciascia S, Cuadrado MJ, Sanna G, Murru V, Roccatello D, Khamashta MA et al (2014) Thrombotic risk assessment in systemic lupus erythematosus: validation of the global antiphospholipid syndrome score in a prospective cohort. Arthritis Care Res 66:1915-1920 